\title{
Development of a Multi-Purpose Easy-to-Use Set of Tools for Home Based Rehabilitation
}

\author{
Use Cases and Applications developed during the REHABitation Project
}

\author{
Veronika David \\ University of Applied Sciences \\ Technikum Wien, Department of \\ Biomedical, Health \& Sports \\ Engineering \\ Wien, Austria \\ veronika.david@technikum-wien.at
}

\author{
Mathias Forjan \\ University of Applied Sciences \\ Technikum Wien, Department of \\ Biomedical, Health \& Sports \\ Engineering \\ Wien, Austria \\ mathias.forjan@technikum-wien.at
}

\author{
Richard Paštěka \\ University of Applied Sciences \\ Technikum Wien, Department of \\ Biomedical, Health \& Sports \\ Engineering \\ Wien, Austria \\ richard.pasteka@technikum-wien.at
}

\author{
Matthias Scherer \\ University of Applied Sciences \\ Technikum Wien, Department of \\ Biomedical, Health \& Sports \\ Engineering \\ Wien, Austria \\ matthias.scherer@technikum-wien.
}

at

\author{
Otto Hofstätter \\ University of Applied Sciences \\ Technikum Wien, Department of \\ Biomedical, Health \& Sports \\ Engineering \\ Wien, Austria
}

otto.hofstaetter@technikum-wien.at

\begin{abstract}
Due to the demographic change, the need of rehabilitation is rising. Home-based rehabilitation can lower the financial burden, support reintegration into daily (work-)life and increase motivation as well as compliance of patients. Several device-supported approaches for rehabilitation were investigated in the research project $\mathrm{REHABi}-$ tation. An insole-based live-feedback system to support patients performing partial weight bearing was developed and tested in a clinical pilot study. Rehabilitative games using commercial gaming control systems like the Microsoft Kinect and the Nintendo Wii Balance Board were developed for range-of-motion and balance training, respectively. For these serious games, usability was tested with the System Usability Scale questionnaire. The comparability of range-of-motion measurements of shoulder movements conducted with inertial measurement units and an optical motion capture system was elaborated. Results fo the clinical study suggest that the patients' compliance with partial weight bearing load restriction was improved with the use of the live-feedback system developed. The use of Wii and Kinect solutions is possible and helps to increase compliance of patients due to high system usability scale scores and positive feedback. The use of intertial measurement units for the detection of motion and its characteristics is highly depending on the used type of system and the intended time span of use. All these approaches were interconnected with diagnosis, corresponding exercises/assessments and tools in the web-based

Permission to make digital or hard copies of part or all of this work for personal or classroom use is granted without fee provided that copies are not made or distributed for profit or commercial advantage and that copies bear this notice and the full citation on the first page. Copyrights for third-party components of this work must be honored.

For all other uses, contact the owner/author(s).

DSAI 2018, fune 20-22, 2018, Thessaloniki, Greece

(C) 2018 Copyright held by the owner/author(s).

ACM ISBN 978-1-4503-6467-6/18/06.

https://doi.org/10.1145/3218585.3218677
\end{abstract}

REHABitation database. It shall be used in future as single point of contact for therapists, patients, manufacturers and interested citizens for preventive and rehabilitative exercise and assessment planning.

\section{CCS CONCEPTS}

- Information systems $\rightarrow$ Data management systems; Users and interactive retrieval; • Applied computing $\rightarrow$ Consumer health; Health informatics;

\section{KEYWORDS}

telerehabilitation, low-cost, eHealth

ACM Reference Format:

Veronika David, Mathias Forjan, Richard Paštěka, Matthias Scherer, and Otto Hofstätter. 2018. Development of a Multi-Purpose Easy-to-Use Set of Tools for Home Based Rehabilitation: Use Cases and Applications developed during the REHABitation Project. In DSAI 2018: 8th International Conference on Software Development and Technologies for Enhancing Accessibility and Fighting Info-exclusion, fune 20-22, 2018, Thessaloniki, Greece. ACM, New York, NY, USA, 8 pages. https://doi.org/10.1145/3218585.3218677

\section{INTRODUCTION}

The participation in social, cultural and occupational areas of life as well as the improvement of Quality of life are major goals of rehabilitation [46]. Whereas patients have a lot of treatments and interventions during their stay in a rehabilitation clinic, this is not the case when they return home. Most of the patients do not perform any type of exercise at home, although rehabilitation process is not completed [16]. Simeone et al. [35] stressed the importance of continuing physical and psychological recovery after discharge. Due to the demographic change, the need of rehabilitation is constantly rising which leads to a financial burden of health care systems [28]. 
Telerehabilitation and rehabilitation at home have the potential to reduce costs and support re-integration in daily life [49]. It has been shown that long stay home care patients receiving rehabilitation at home have improved outcomes and need fewer costly health services [9]. However, in most cases, patients receive rehabilitative exercises instructions for home verbally or in the form of paper copies. In a home setting, neither the compliance of patients nor the correct performance of these exercises can be checked, as there are hardly any tools or interventions to support the patient. Thus, the interest for home-based rehabilitation tools, which helps the patient to keep rehabilitation exercises and to increase motivation, is growing. These tools should be easy-to-use, easy available, light weighted if worn on the body, and inexpensive. Therefore, to further reduce costs for rehabilitation applications, the approach to use off-the-shelf products or gaming systems has arisen in the last years. [16]

The research project REHABitation deals with the development of technology-based rehabilitative and preventive measures for the home environment. One focus is the realisation of these measures with off-the-shelf devices, which are inexpensive and easily available. Thus, a central question is the comparability of parameters from home based tools and clinical diagnosis tools. In course of this project several usecases were developed as well as an overview database for the interconnection of rehabilitative exercises and available tools were created. In the present work, three of the usecases as well as the implementation of the REHABitation database are presented.

The approaches include partial weight bearing after injuries of the lower extremities or total hip arthroplasty (THA) using a livefeedback system, training of movement and coordination of upper extremities for neurological patients based on Microsoft Kinect and training of coordination and balance with a Nintendo Wii Balance Board. Further on, the comparability of Inertial Measurement Units (IMUs) to a gold-standard motion analysis system was elaborated, as the use of IMUs in motion analysis is on the rise, but data quality is highly dependent on data processing and handling [37]. The suitable devices and interoperable tools which were researched during the project as well as all approaches developed, were entered into a database, which also include the interconnection of these tools with rehabilitative exercises and fields of application.

\subsection{State of the Art}

After lower limb injuries or surgical replacement of hip or knee joints, a common rehabilitative measure is partial weight bearing (PWB)[2,34]. This measure proved to be beneficial of the healing process after hip operations, knee surgeries, and fractures in or around the joints of the lower limbs [8]. PWB allows an optimized rehabilitation process with reduces healing time and quicker reestablishment of normal gait [20] The number of total hip arthroplasties (THA) has risen since the year 2000 and countries like Germany and Austria show very high incidence rates of 286-306 cases per 100.000 population [29]. In most cases, the partial load is trained statically with a scale or force plate [24, 48] making it very difficult for patients to translate the static trained partial load to dynamic movements. This leads to almost $90 \%$ of patients exceeding the prescribed load significantly while walking [38, 45].
Besides static training, also verbal instructions lead to the same poor outcome in regard to the ability of patients reproducing the prescribed partial load [19, 41, 42]. An approach to improve compliance with PWB, which already showed its positive effects in clinical environment and rehabilitation centres, is to provide the patient with biofeedback. Nevertheless, most studies agreed that it is even more important for the success of the PWB method to give patients the possibility to monitor and quantify their loading outside of a clinical environment in daily life, as the largest part of rehabilitation occurs after discharge from the clinic $[18,19]$.

This applies also for stroke patient, for which it is very important to continue physical and psychological recovery when they return home [35]. In high income countries, stroke is the leading cause of death and second leading cause of disability [25,44]. More than $50 \%$ of the stroke patients surviving the acute phase of stroke, lose their ability to walk[13, 43]. Cameirao et al. [7] described ICT (Information and Communication Technology) home-based solutions as strategy to allow patients tu pursue their treatments without institutionalization. They presented home-based rehabilitation exercises with a Microsoft Kinect for movement tracking and a Virtual Coach. Faria et al. [17] developed a Rehab@home system composed of two Wii balance boards and a webcam for neurological patients like stroke survivors. Telerehabilitation and rehabilitation with low-cost consumer technologies seems to be a solution to increase rehabilitation access [14]. Commercial gaming systems, like the Nintendo Wii Balance Board or the Microsoft Kinect, are more and more intensively used for rehabilitation purposes due to their low-costs and easy availability $[14,16]$. In connection with virtual reality, which is reported to increase motivation and engagement of patient performing rehabilitative exercises [26], custom made applications with conventional PCs and commercial gaming systems seems promising solutions for rehabilitation at home [16].

Inertial measurement units (IMU), a combination of accelerometers and gyroscopes, have become widely used in the research of wearable motion analysis in the last years, due to rapid development of microelectromechanical systems [37]. The IMUs have several advantages such as lightweight, small size, low power consumption, portability and low-cost [15, 37]. They are used as an alternative to laboratory-based systems to gather motion data or evaluate postural control and balance [30,47]. Thus, they allow monitoring motion in real-life environments and seems therefore suitable for applications in home rehabilitation. Although some studies showed good agreement of IMU measurements with clinical systems [5], the quantification of gait by using IMUs have not yet been driving changes in clincical practice as outcomes measurements are highly heterogeneous [15]. Sprager and Juric [37] conclude that IMU-based motion analysis shows great potential, but needs to cope with several limitations like reproducable sensor positioning, data connectivity, data handling and processing.

The research questions focused on the actual applicability of lowcost and commercially available system for clinical and home-based rehabilitation purposes in comparison to state-of-the-art solutions. 


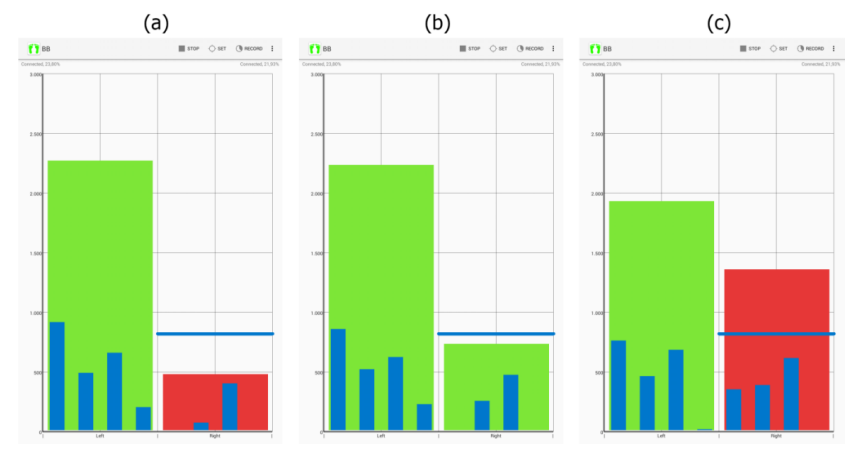

Figure 1: Screenshots of the live-feedback Android application. The overall load for left and right, respectively, is shown as big green bar, whereas the small bars represent the pressure from the single pressure sensors (four per insole). Load restriction is set for the right leg, the threshold is marked with the horizontal blue line. (a) and (c) show incorrectly loaded steps where the bar turns red, in (c) the threshold feedback is triggered (sounds and/or vibration). (b) correctly loaded step [39].

\section{METHODS}

\subsection{Feedback system for partial weight bearing}

The live-feedback system for PWB consists of the multimodal, mobile gait analysis system eSHOE, which was developed by the Central European Institute of Technology (CEIT RALTEC, Schwechat) in collaboration with the University of Applied Sciences Technikum Wien [11, 21-23] and an Android-application [39, 40]. The eSHOE system are orthopaedic insoles with different sensor embedded, capable of measuring pressure under four specific areas under the foot, 3D-acceleration as well as 3D angular velocity, available in sizes EUR36-EUR44. The insoles were validated against golden standard systems where they results showed strong linear relationship and good agreement $[10,12]$. For the intended use in PWB, only the pressure data are of interest, which are recorded via four A401 Tekscan sensitive resistor sensors (FSR) under the heel, the first and fifth metatarsal heads and the big toe. The system transmits sensor data via Bluetooth using a KC22.6, KC Wirefree module to the PWB Android application. In the application, pressure data can be either recorded or visualized as bar charts which proved to be easy understandable for patients. An individual threshold value can be set by the medical professional and the form of the feedback, either visual, haptic, acoustic or a combination of these, can be configured which is triggered whenever the set threshold is reached.

Ten geriatric patients (median age 78; median BMI 22.1; 8f/2m) participated in a clinical pilot study during their inpatient stay. On four measurement dates the participants walked in a straight line with the inserted insoles once without feedback function and once with activated feedback function of the live-feedback system. Parameters like correctly loaded steps and mean maximum load were extracted from the data gathered and compared. A step is correctly loaded if the load is in the range of the prescribed load
1 I think I would like to use this system frequently
2 I think I would need the support of a technical person to be able to use this system
3 I would imagine that most people would learn to use this system very quickly.
4 I felt very confident using the system
5 I needed to learn a lot of things before I could get going with this system.
6 The task was entertaining.
7 The task was too long.
8 The task was easy to understand.
9 It was difficult to control the virtual hands
10 I would like to continue this treatment.

Table 1: Question of the modified System Usability Scale (mSUS), based on [7]

$\pm 10 \%$ of bodyweight. The significance of the results was tested with Wilcoxon-Rank-Sum and Signed-Rank-Tests.

\subsection{Rehabilitative game for upper extremities}

The rehabilitative game for the upper extremities of neurological patients was developed based on requirements defined by therapists from the Neurological Rehabilitation Centre Rosenhügel (NRZ Rosenhügel, Vienna). One important requirement was the adaptability of the rehabilitative game to the patients' needs and abilities, which could change from day to day. The game itself was implemented in C\# and UnityScript, runs on a standard personal PC or laptop and includes two different life-near training scenes with different levels of difficulty. These training scenes are a book shelf, where the user has to prevent them from falling out of the shelf, and a kitchen scene, where the user has to grab the right object which is textual given. Thus, the kitchen scene also trains cognitive functions, as the written word has to be recognized and associate with the right object. The games are controlled by hand movements which are detected via the Microsoft Kinect 2 . The virtual scenes are either displayed on a big screen or projected to the wall via video projector. The therapist can supervise the movement while standing behind the patient as the program just tracks the hands of the patient (see Figure 2). Virtual hands, which are positioned according to the detected coordinates of the hand joints, are overlayed in the scenes. At the NRZ eleven stroke patients (average age $58,9 \mathrm{~m} / 2 \mathrm{f}$ ) took part in a usability study. Over one week the patients performed the exercises of the game at least twice. Afterwards they filled out a modified system usability scale (mSUS) questionnaire (see Table 1), which is based on the standardized SUS [6]. Five questions were changed concordant with [7] to fit the setting, as some questions were not answerable by the patients. The mSUS score results in values between 0 and 100 without unit, while 100 represents high usability. Five therapist which took part in this usability study filled out the standard SUS. 

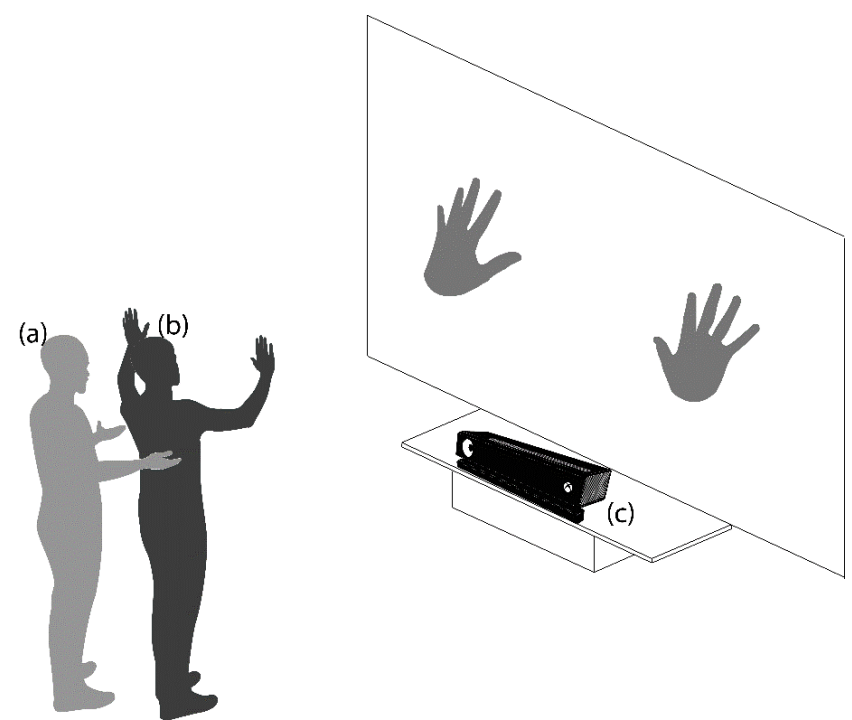

Figure 2: Basic exercise setup for upper extremities: The therapist (a) can supervise of the patient (b) while the Kinect (c) tracks the hand movement [33].

\subsection{Rehabilitative game for balance training}

Another rehabilitative game for balance training using the Nintendo Wii Balance Board was developed in cooperation with the NRZ Rosenhügel. Again, requirements from the therapists were collected which leads to exercises adaptable to the patients' abilities and needs. Different balance exercises were realised, like a virtual kitchen, a parachute jump and dancing the Waltz. Like the rehabilitation game for upper extremities, this balance game was implemented in C\# and UnityScript, but needs to run on a personal computer or laptop which provides at least Bluetooth 2.3 interface. The user controls a virtual avatar with the Wii Balance Board by shifting his body weight. The therapist can supervise the movements of the patient while the patient is secured with a safety harness ((c) in Figure 3). 23 neurological patients $(60.3 \pm 16.9$ yrs, $14 \mathrm{~m}, 9 \mathrm{f})$ with different clinical conditions, who got balance training prescribed during their stay in the rehabilitation clinics, took part in a usability study. They performed the balance exercises and filled out the SUS questionnaire [6].

\subsection{Validation of interial measurment units}

To compare IMUs with gold-standard motion analysis systems, Range of Motion (ROM) measurements of the shoulder joints were conducted with IMUs and a Vicon Motion Analysis system simultaneously. Three different shoulder movements, namely shoulder abduction, external rotation of shoulder joint (from neutral position) and horizontal adduction were performed 35 times. The measurements were conducted with (prototypical) inertial measurement units from the ETH Zurich consisting of a microcontroller, an IMU-module MPU-9150 (InvenSense), a standard accelerometer, an accumulator, a SD-memory-card, a micro USB interfaces and a Bluetooth interface. The Vicon Motion Analysis system was set up with seven Vicon Bonita infrared cameras mounted in a circular

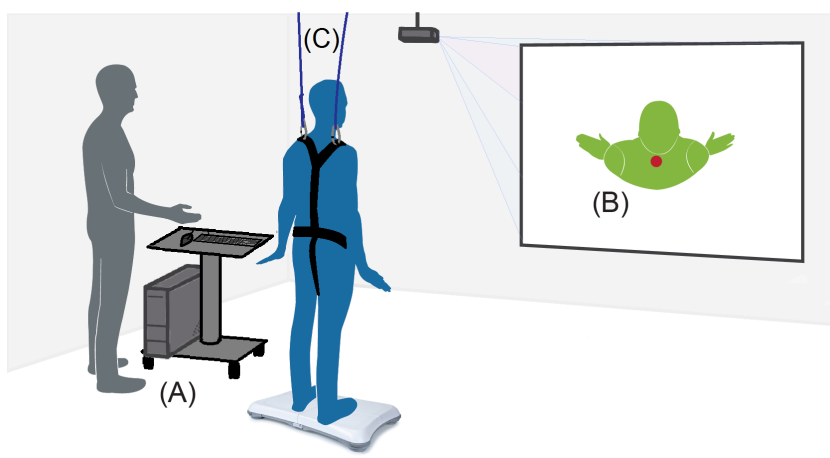

Figure 3: Basis balance exercise setup: The patient is standing on the Wii balance board while secured in a safety harenss (c). The therapist sets the parameters for the exercise and operates the software on the PC station (a). (b) is the projection of the avatar on the wall or screen with labeling of the COP as dot [27].
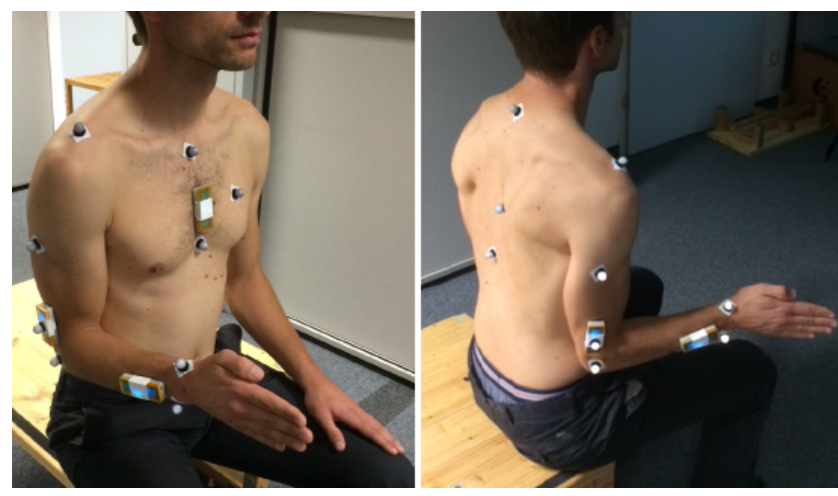

Figure 4: Placement of IMUs and marker setup for shoulder movement measurements [36].

way around the measurement area. The marker setup is based on the Plug-In-Gait Marker Model [29] and the IMUs were placed on the sternum, upper and lower arm (Figure 4). Agreement between the ROM-measurements were compared by Bland Altman plots $[1,4,32]$ and quantified by the Root Mean Square (RMS) as well as with Pearson's correlation coefficient (r) for the values of the range as well as for peak values.

\subsection{Exercise and assessment database}

Exercises and assessments from international rehabilitation sources as well as preventive and sports oriented focuses were integrated into the developed web based easy-to-use information database for assessments, exercises and tools [31]. The database is structured to allow usage by any involved user. This means that the therapist, as the first medical professional in a typical rehabilitation process may search for suitable assessment methods and exercises for the individual patient. These may be linked with tools available in the database. The patient is handed over a collection and description of the selected exercises. The patient can use this rudimentary information and look up more specific information in the database 


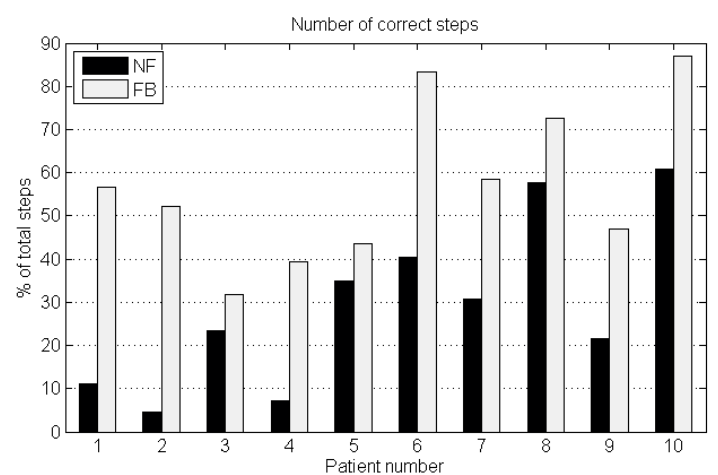

Figure 5: Number of correctly loaded steps in \% of total steps without feedback function (NF, black) and with feedback function (FB, grey) of the live-feedback system developed [39].

and can also watch tutorial videos or graphical representations for better understanding. The third possible user group of the database are device manufacturers, who can enter their devices according to their own information into the database. The implemented authentication methods prevents the database from misuse of unauthorised change of datasets. As a single point of contact the database includes a single easy-to-use search field, which allows search queries by each character. Information about all matching results will be displayed immediately and further information can be retrieved by a single click.

\section{RESULTS}

PWB performance of all patients improved with the use of the livefeedback system (see Figure 5), the average improvement of the number of correctly loaded steps was $27.9 \%(\mathrm{p}=0.002)$. Additionally, the maximal load decreased in 9 out of 10 patients and in average from 31.5 to $21.6 \%(\mathrm{p}=0.006)$ difference to the prescribed load (Figure 6).

The virtual rehabilitation game for upper extremities was implemented with two different exercises, bookshelf and kitchen scene, with ten levels of difficulty and with a training as well as a comparison mode. When using the system the first time, a calibration is done for each patient to determine the individual maximal ROM to ensure that with a calibration factor the patient is still able to reach with the virtual hands all corners of the screen. In the exercise 'bookshelf' the task is to prevent books from falling out from the book shelf, where caught books move back to their position in the shelf and dropping books disappear (Figure 7). In the exercise 'kitchen environment' the name of an object is displayed which has to be recognized, grabbed and put into the middle of the kitchen counter before a time defined has elapsed (Figure 8).

The results of the mSUS filled out by the patients was $78.4 \pm 11.1$ whereas the results of the SUS filled out by the therapists was $91.0 \pm 4.9$. The mSUS value is in the range of a 'good' usability and the SUS value is in the range of an 'excellent' usability, according to Bangor et al. [3].

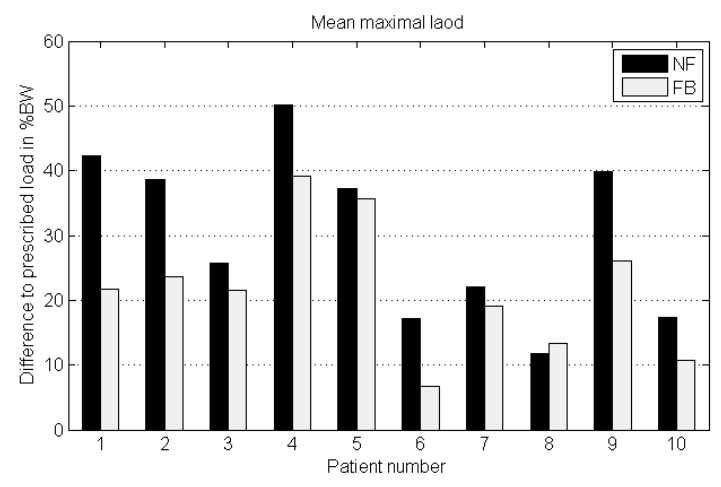

Figure 6: Maximal load (expressed as difference to the prescribed partial load in \% of body weight) without (black) and with (grey) feedback function of the live-feedback system developed. 9 out of 10 patients could reduce the maximal load with the help of the system [39].

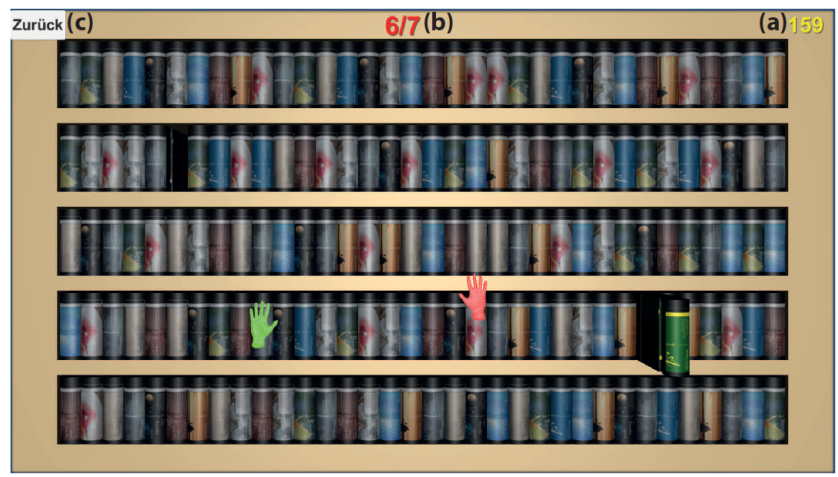

Figure 7: Screenshot of the 'bookshelf' exercise for training of the upper extremities. (a) shows the remaining time in seconds, (b) displays the books caught, and (c) enables the return to the home screen [33].
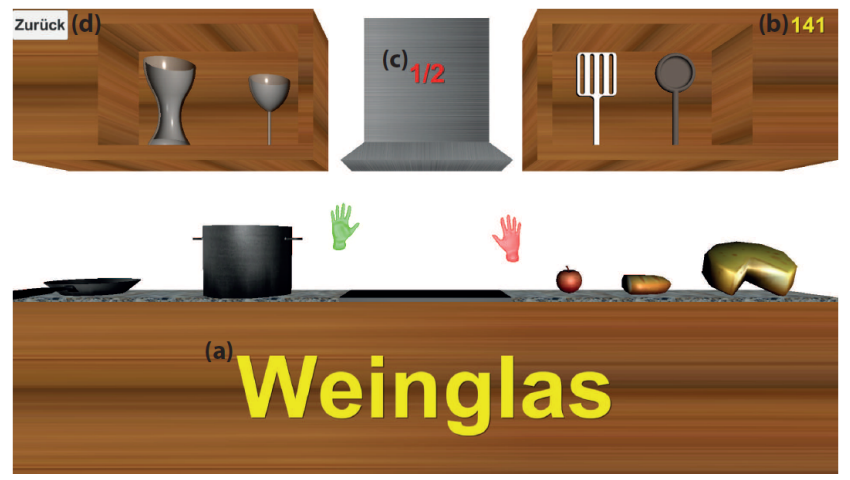

Figure 8: Screenshot of the 'kitchen environment' where (a) displays the target object, (b) shows the remaining time in seconds, (c) stands for the objects recognized, and (d) enables the return to the home screen [33]. 


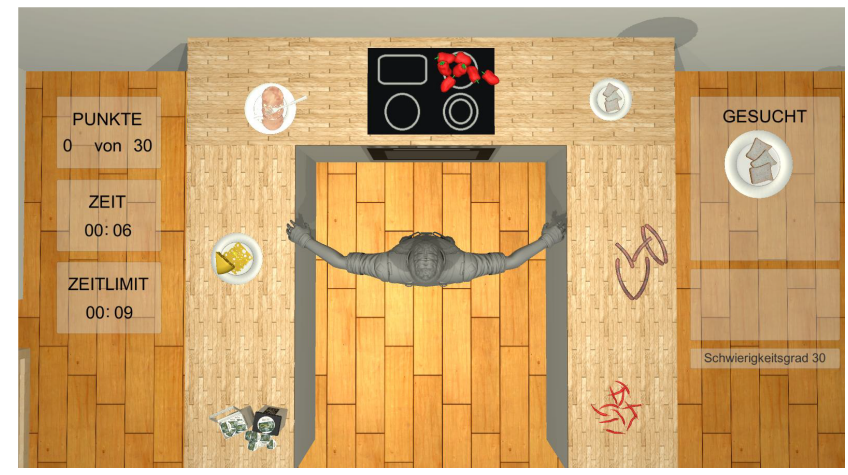

Figure 9: Screenshot of the virtual kitchen for balance training, the avatar moves based to the COP measured. In the right upper corner the ingredient which as to be acquired and streched for is displayed [27].

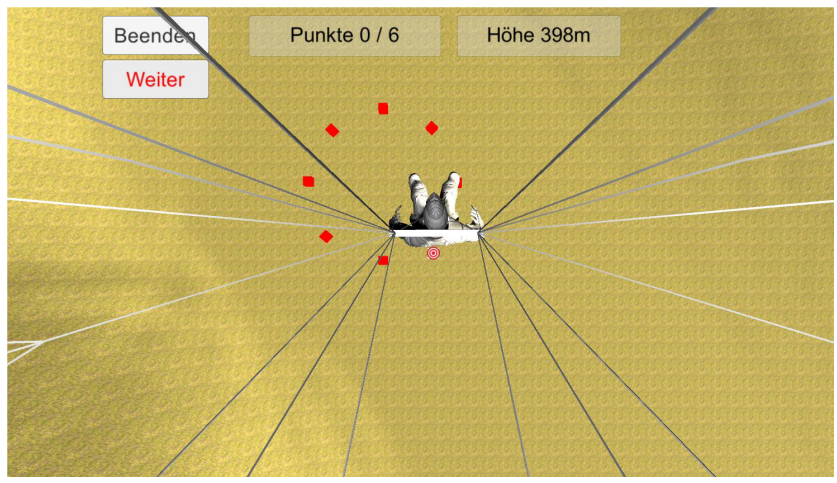

Figure 10: Screenshot of the parachute balance exercise where the avatar has to be navigated trough intermediate goals, indicated as a circle of red dots. The directions of the avatar are controlled with weightshifting on the Wii balance board [27].

The balance training game using the Nintendo Wii Balance Board includes four different exercises with up to 30 difficulty levels. As examples, the screenshot of the virtual kitchen (Figure 9) and the parachute jump can be seen in Figure 10. In the virtual kitchen the patient has to shift its weight in the direction of the ingredient which has to be acquired, indicated by the picture displayed in the right upper corner of the screen. For the parachute jump, the goal is to maneuvre the chute through intermediate goals, indicated with red circles until it should be landed in the middle of the end goal, displayed as common target with red and with circles. The avatar is controlled via weight shifting on the Wii Balance Board and moves accordingly to the progress of centre-of-pressure (COP) gathered in the virtual scenes.

The patients rated the overall balance training game with 85 , the virtual kitchen was rated with 81.25 and the parachute exercise with 100 points in the SUS scale. The value from the virtual kitchen is in the range of 'good' usability, whereas the overall value of 85 and

\begin{tabular}{llll}
\hline \multicolumn{4}{c}{ Range values } \\
$\begin{array}{l}\text { Shoulder } \\
\text { movement }\end{array}$ & abduction & $\begin{array}{l}\text { external rota- } \\
\text { tion }\end{array}$ & $\begin{array}{l}\text { horizontal ad- } \\
\text { duction }\end{array}$ \\
\hline $\begin{array}{l}\text { Pearson's r } \\
\text { RMS }\end{array}$ & 0.30 & 0.83 & 0.59 \\
\hline & $8.87^{\circ}$ & $13.28^{\circ}$ & $5.51^{\circ}$ \\
Shoulder & abduction & $\begin{array}{l}\text { Peak values } \\
\text { external rota- }\end{array}$ & $\begin{array}{l}\text { horizontal ad- } \\
\text { dion }\end{array}$ \\
\hline $\begin{array}{l}\text { Pearson's r } \\
\text { RMS }\end{array}$ & 0.34 & 0.65 & 0.12 \\
\hline
\end{tabular}

Table 2: Range- and peak value results for the agreement between IMU and optical motion analysis system for the shoulder movements of abduction, external rotation and horizontal adduction using Pearson's $r$ and RMS

100 for the parachute jump is in the range of 'excellent' usability, according to Bangor et. al [3].

Comparison of the results of ROM measurements with the IMUs and the optical motion analysis system resulted in Pearson's $r$ of $0.3-0.83$ and a RMS of $8.87^{\circ}-13.28^{\circ}$ for the range and in Pearson's $r$ of $0.12-0.65$ or $12.69^{\circ}-15.01^{\circ}$, respectively, for the peak values (see Table 2). Although most values in the Bland-Altman plot (Figure 11) lie in the limits of agreement $\left(2^{*} \mathrm{SD}\right)$, the plots show poor agreement, as the mean differences are between $4.73^{\circ}$ and $13.21^{\circ}$ for shoulder movements. These limits of agreement are clinically not acceptable.

At the current point in time, the REHABitation database (see Figure 12) includes 450 exercises and assessments. These are linked to supporting tools and/or software solutions (equipment). All entries are therefore directly connected to a diagnosis - thereby to a ICD code - and an area of assessment. The database allows by its setup users of different views, like patients, medical professionals, therapists and even equipment manufacturers to access the exercises and assessments, search for them and introduce new combinations, if desired. Therefore a therapist can provide an individual combination of each patient. This web based plattform is designed to be used as interactive and easy-to-use as possible and therefore also includes live search, performed during typing of each character.

\section{DISCUSSION}

Results of the clinical pilot study suggest that the patients' compliance with PWB load restriction was improved with the use of the live-feedback system developed. The number of correctly loaded steps increased for all patients with the help of the feedback. The changes were statically significant, what leads to the assumption that the system is applicable for supporting patient with the performance of PWB. Additionally, the system is unobtrusive as the instrumented insoles are based on standard orthopaedic insoles and data are wirelessly transmitted to the Android-application, which makes it suitable for the use outside of clinical environments. The use of Wii and Kinect solutions is possible and helps to increase 


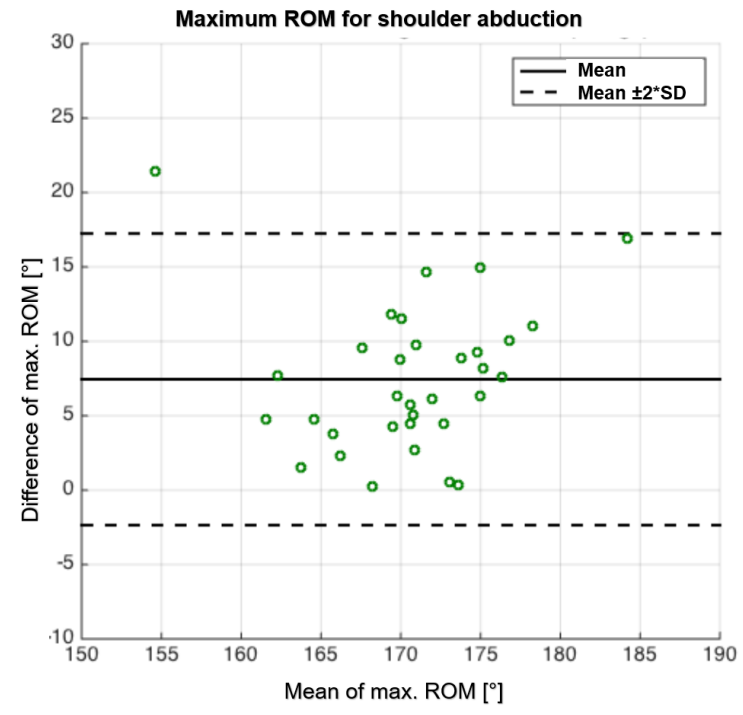

Figure 11: Bland-Altman plot for the maximum ROM value during shoulder abduction gathered with IMUs and the optical motion analysis system. The mean difference is $7.45^{\circ} \pm 4.90^{\circ}[36]$.

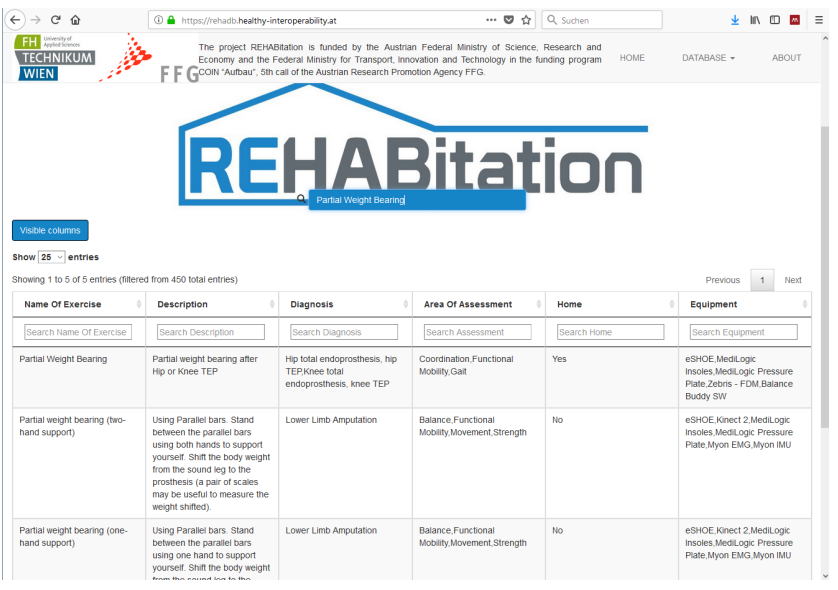

Figure 12: Screenshot of the REHABitation database.

compliance of patients due to high SUS scores and positive feedback. Due to their low-costs and easy availability, it is suitable for the use at home. Nevertheless, these solutions are not certified as medical products and can therefore not be included into the clinical routine, or even prescribed as training equipment. The agreement of shoulder ROM measurements with IMUs and a optical motion capture system was poor in contrast to the results of [5] who demonstrated good agreement between IMU and an optoelectronic motion capture system for pelvic orientation. The use of IMUs for the detection of motion and its characteristics is highly depending on the used type of system and the intended time span of use. Nevertheless, IMUs can be used for simple monitoring activities as they are mostly easy attachable and not complex in handling. Considering the current number of users, the performance of the REHABitation database seem to be sufficient, but will have to be adapted with increasing number of users and service requests. However, the system has to be tested under high load and stress for proving its daily-use capabilities. Additionally, this web based solution still has to be improved for accessibility according to gold standards and W3C conventions. Hence, the database can be used in future as single point of contact for therapists, patients, manufacturers and simply interested citizens for preventive and rehabilitative exercise and assessment planning.

\section{LIMITATIONS}

The insole system is the only development which was validated in course of former projects, the other developments like the rehabilitative games, were developed during the project REHABitation and thus, the first step was to evaluate usability of the systems before efficiency has to be tested in clinical studies. For the shoulder movements the investigation presented here aimed to compare IMU movement data with a golden-standard movement analysis system as many IMUs systems are currently on the market for upper extremity rehabilitation although the accuracy is not proven. The studies described in the paper have clear limitation as the number of participants was low and only specific pathologies were examined. Thus, in future studies the provided solutions should be tested with broader clinical pictures and an appropriate number of participants.

\section{ACKNOWLEDGMENTS}

The authors thank the clinical partners from the Sophienspital and NRZ Rosenhügel as well as all colleagues from the REHABitation project of the UAS Technikum Wien for their support during the research process. The project REHABitation is funded by the Austrian Research Promotion Agency FFG.

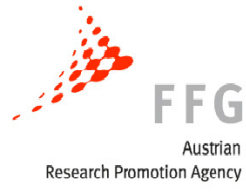

\section{REFERENCES}

[1] D.G. Altman and J.M. Bland. 1983. Measurement in Medicine: the Analysis of Method Comparison Studies. The Statistician 32 (1983), 307-317.

[2] Alicia Bailón-Plaza and Marjolein C.H. Van Der Meulen. 2003. Beneficial effects of moderate, early loading and adverse effects of delayed or excessive loading on bone healing. Journal of Biomechanics 36, 8 (2003), 1069-1077. https://doi.org/ 10.1016/S0021-9290(03)00117-9

[3] Aaron Bangor, Philip Kortum, and James Miller. 2009. Determining what individual SUS scores mean: Adding an adjective rating scale. Fournal of usability studies 4, 3 (2009), 114-123. https://doi.org/66.39.39.113 arXiv:978-0-9846455702-0-1

[4] J.M. Bland and D.G. Altman. 1986. Statistical Methods for Assessing Agreement Between Two Methods of Clinical Measurement. Lancet 327, 8476 (feb 1986), 307-310. https://doi.org/10.1016/S0140-6736(86)90837-8

[5] S. A.A.N. Bolink, H Naisas, R Senden, H Essers, I C Heyligers, K Meijer, and B Grimm. 2016. Validity of an inertial measurement unit to assess pelvic orientation angles during gait, sit-stand transfers and step-up transfers: Comparison with an optoelectronic motion capture system. Medical Engineering and Physics 38, 3 (2016), 225-231. https://doi.org/10.1016/j.medengphy.2015.11.009

[6] John Brooke. 1996. System Usability Scale (SUS): A Quick-and-Dirty Method of System Evaluation User Information. Usability Evaluation In Industry (1996). https://doi.org/10.1002/hbm.20701

[7] Mánica S. Cameirão, Sergi Bermúdez I. Badia, Esther Duarte Oller, and Paul FMJ Verschure. 2010. Neurorehabilitation using the virtual reality based Rehabilitation Gaming System: Methodology, design, psychometrics, usability and validation. Journal of NeuroEngineering and Rehabilitation 7, 1 (sep 2010), 48. https://doi. org/10.1186/1743-0003-7-48

[8] D H K Chow and C T K Cheng. 2000. Quantitative analysis of the effects of audio biofeedback on weight-bearing characteristics of persons 
with transtibial amputation during early prosthetic ambulation. four nal of Rehabilitation Research and Development 37, 3 (2000), 255-260. http://www.ncbi.nlm.nih.gov/pubmed/10917257http://ovidsp.ovid.com/ ovidweb.cgi? $\mathrm{T}=\mathrm{JS}\{\&\} \mathrm{CSC}=\mathrm{Y}\{\&\} \mathrm{NEWS}=\mathrm{N}\{\&\} \mathrm{PAGE}=$ fulltext $\{\&\} \mathrm{D}=$ emed $8\{\&\} \mathrm{AN}=$ 30641222http://bf4dv7zn3u.search.serialssolutions.com.myaccess.library. utoronto.ca/?url\{_\}ver=Z39.88-2004\{\&\}rft\{_\}val\{_\}fmt=info:ofi/fmt:kev

[9] Richard J. Cook, Katherine Berg, Ker Ai Lee, Jeffrey W. Poss, John P. Hirdes, and Paul Stolee. 2013. Rehabilitation in home care is associated with functional improvement and preferred discharge. Archives of Physical Medicine and Rehabilitation 94, 6 (2013), 1038-1047. https://doi.org/10.1016/j.apmr.2012.12.024

[10] Veronika David. 2012. Development of Validation Methods for the Mobile Gait and Motion Pattern Analysis System vitaliSHOE. Master Thesis. University of Applied Sciences Technikum Wien.

[11] V. David, M. Forjan, J. Martinek, S. Kotzian, H. Jagos, and D. Rafolt. 2017. Evaluating wearable multimodal sensor insoles for motion-pattern measurements in stroke rehabilitation - A pilot study. In IEEE International Conference on Rehabilitation Robotics, Vol. 2017. IEEE, 1543-1548. https://doi.org/10.1109/ICORR.2017. 8009467

[12] V. David, H. Jagos, S. Litzenberger, and M. Reichel. 2012. Instrumented insole for mobile and long distance motion pattern measurement. Procedia Engineering 34 (2012), 760-765. https://doi.org/10.1016/j.proeng.2012.04.130

[13] P. W. Duncan, R. Zorowitz, B. Bates, J. Y. Choi, J. J. Glasberg, G. D. Graham, R. C. Katz, K. Lamberty, and D. Reker. 2005. Management of Adult Stroke Rehabilitation Care: A Clinical Practice Guideline. Stroke 36, 9 (2005), e100-e143. https: //doi.org/10.1161/01.STR.0000180861.54180.FF

[14] Mary C Edgar, Sarah Monsees, Josina Rhebergen, Jennifer Waring, Todd Van der Star, Janice J. Eng, and Brodie M. Sakakibara. 2017. Telerehabilitation in Stroke Recovery: A Survey on Access and Willingness to Use Low-Cost Consumer Technologies. Telemedicine and e-Health 23, 5 (2017), 421-429. https://doi.org/ 10.1089/tmj.2016.0129

[15] Miguel Escudero, Roman Schniepp, Alvaro Sanchez-Ferro, Aliénor Vienne Rémi P Barrois, Stéphane Buffat, Damien Ricard, and Pierre-Paul Vidal. 2017. Inertial Sensors to Assess Gait Quality in Patients with Neurological Disorders: A Systematic Review of Technical and Analytical Challenges. (2017) https://doi.org/10.3389/fpsyg.2017.00817

[16] Carlos Faria, Jorge Silva, and Aurélio Campilho. 2015. Rehab@home: a tool for home-based motor function rehabilitation. Disability and Rehabilitation: Assistive Technology 10, 1 (2015), 67-74. https://doi.org/10.3109/17483107.2013.839749

[17] Carlos Faria, Jorge Silva, and Aurélio Campilho. 2015. Rehab@home: A tool for home-based motor function rehabilitation. Disability and Rehabilitation: Assistive Technology 10, 1 (2015), 67-74. https://doi.org/10.3109/17483107.2013.839749

[18] Michael C Fu, Levi DeLuke, Rafael A Buerba, Richard E Fan, Ying Jean Zheng Michael P Leslie, Michael R Baumgaertner, and Jonathan N Grauer. 2014. Haptic Biofeedback for Improving Compliance With Lower-Extremity Partial Weight Bearing. Orthopedics 37, 11 (nov 2014), e993-e998. https://doi.org/10.3928/ 01477447-20141023-56

[19] Joshua W Hustedt, Daniel J Blizzard, Michael R Baumgaertner, Michael P Leslie, and Jonathan N Grauer. 2012. Current advances in training orthopaedic patients to comply with partial weight-bearing instructions. (mar 2012), 119-125 pages. http://www.ncbi.nlm.nih.gov/pubmed/22461750http://www. pubmedcentral.nih.gov/articlerender.fcgi?artid=PMC3313526

[20] E Isakov. 2007. Gait rehabilitation: a new biofeedback device for monitoring and enhancing weight-bearing over the affected lower limb. Europa Medicophysica 43, 1 (mar 2007), 21-26. https://doi.org/R33061641[pii]

[21] Harald Jagos. 2016. Mobile Gait Analysis via instrumented Shoe Insoles - eSHOE. Dissertation. Technical University Vienna.

[22] Harald Jagos, Veronika David, Michael Haller, Stefan Kotzian, Manuela Hofmann, S. Schlossarek, Karolina Eichholzer, Manfred Winkler, Matthias Frohner, Martin Reichel, Winfried Mayr, and Dietmar Rafolt. 2015. A Framework for (Tele-) Monitoring of the Rehabilitation Progress in Stroke Patients. Applied Clinical Informatics 6, 4 (2015), 757-768. https://doi.org/10.4338/ACI-2015-03-RA-0034

[23] H. Jagos, J. Oberzaucher, M. Reichel, W. L. Zagler, and W. Hlauschek. 2010. A Multimodal Approach for Insole Motion Measurement and Analysis. Procedia Engineering - The engineering of sport 800 (2010), 1-6.

[24] I. Klöpfer-Krämer and P. Augat. 2010. Teilbelastung in der Rehabilitation. Der Unfallchirurg 113, 1 (2010), 14-20. https://doi.org/10.1007/s00113-009-1717-8

[25] Peter Langhorne, Fiona Coupar, and Alex Pollock. 2009. Motor recovery after stroke: a systematic review. The Lancet Neurology 8 (2009), 741-754. https //doi.org/10.1016/S1474-4422(09)70150-4 arXiv:10.1016/S1474-4422(09)70150-4.

[26] Gwyn N Lewis, Claire Woods, Juliet A Rosie, and Kathryn M McPherson. 2011 Virtual reality games for rehabilitation: Perspectives from the users and new directions. In International Conference on Virtual Rehabilitation. 1-2. https: //doi.org/10.1109/ICVR.2011.5971842

[27] Michael Mörtenhuber. 2017. Development and Usability Verification of a Virtual Reality Balance Improvement System for Neurological Rehabilitation with the Wi Balance Board. Master Thesis. University of Applied Sciences Technikum Wien.

[28] R Müller and A Klimesch. 2011. Entwicklung der ambulanten medizinischen Rehabilitation in Österreich. In Reha-Bericht 2011. Pensionsversicherungsanstalt,
Vienna, 26-30.

[29] C. Pabinger and A Geissler. 2014. Utilization rates of hip arthroplasty in OECD countries. Osteoarthritis and Cartilage 22, 6 (2014), 734-741. https://doi.org/10. 1016/j.joca.2014.04.009

[30] Enrica Papi, Denise Osei-Kuffour, Yen-Ming A Chen, and Alison H McGregor. 2015. Use of wearable technology for performance assessment: A validation study. Medical Engineering \& Physics 37, 7 (jul 2015), 698-704. https://doi.org/ 10.1016/j.medengphy.2015.03.017

[31] Richard Pašteka, Mathias Forjan, and Stefan Sauermann. 2017. Development of a multidisciplinary and telemedicine focused system database. Studies in Health Technology and Informatics 236 (2017), 144-151. https://doi.org/10.3233/ 978-1-61499-759-7-144

[32] Peter Rehak. 1998. Auswertung und Präsentation experimenteller Daten. Acta Chirurgica Austriaca 30, 147 (1998), 29-32.

[33] Bernhard Riess. 2017. Development and Feasibility Assessment of a System for Neurological Rehabilitation using Kinect v2. Master Thesis. University of Applied Sciences Technikum Wien.

[34] AlsayedA Shanb and EnasF Youssef. 2014. The impact of adding weight-bearing exercise versus nonweight bearing programs to the medical treatment of elderly patients with osteoporosis. Fournal of Family and Community Medicine 21, 3 (2014), 176. https://doi.org/10.4103/2230-8229.142972

[35] Silvio Simeone, Serenella Savini, Marlene Z. Cohen, Rosaria Alvaro, and Ercole Vellone. 2015. The experience of stroke survivors three months after being discharged home: A phenomenological investigation. European fournal of Cardiovascular Nursing 14, 2 (2015), 162-169. https://doi.org/10.1177/1474515114522886

[36] Christoph Smrz. 2016. Evaluierung der Genauigkeit eines Intertialsensorsystems bei Bewegungsumfangstests der Schulter. Master Thesis. UAS Technikum Wien.

[37] Sebastijan Sprager and Matjaz B Juric. 2015. Inertial sensor-based gait recognition: A review. Vol. 15. 22089-22127 pages. https://doi.org/10.3390/s150922089 arXiv: 1708.09366

[38] R. Stangl, R. Krug, F. F. Hennig, and J. Gusinde. 2004. Kontrollierte teilbelastung unter verwendung einer biofeedbackschuhsohle. Unfallchirurg 107, 12 (dec 2004), 1162-1168. https://doi.org/10.1007/s00113-004-0819-6

[39] Andrés Igor Tkachenko Bril. 2017. Evaluation of the feasibility and acceptance of a partial weight-bearing supporting biofeedback system. Masters Thesis. University of Applied Sciences Technikum Wien.

[40] A. I. Tkachenko Bril, V. David, M. Scherer, H. Jagos, P. Kafka, and A. Sabo. 2016. Development of a Wearable Live-feedback System to Support Partial Weightbearing while Recovering from Lower Extremity Injuries. In Procedia Engineering, Vol. 147. 157-162. https://doi.org/10.1016/j.proeng.2016.06.206

[41] M Tveit and J Kärrholm. 2001. Low effectiveness of prescribed partial weight bearing. Continuous recording of vertical loads using a new pressure-sensitive insole. Fournal of rehabilitation medicine : official journal of the UEMS European Board of Physical and Rehabilitation Medicine 33, 1 (jan 2001), 42-46. http: //www.ncbi.nlm.nih.gov/pubmed/11480470

[42] Attila Vasarhelyi, Tobias Baumert, Christoph Fritsch, Werner Hopfenmüller, Georg Gradl, and Thomas Mittlmeier. 2006. Partial weight bearing after surgery for fractures of the lower extremity - Is it achievable? Gait and Posture 23, 1 (jan 2006), 99-105. https://doi.org/10.1016/j.gaitpost.2004.12.005

[43] Martha Visintin, Hugues Barbeau, Nicol Korner-bitensky, and Nancy E Mayo. 1998. A New Approach to Retrain Gait in Stroke Patients Through Body Weight Support and Treadmill Stimulation Martha Visintin, Hugues Barbeau, Nicol Korner-Bitensky and Nancy E. Mayo Stroke 1998;29;1122-1128. Stroke 29, 6 (1998), 1122-1128. https://doi.org/10.1161/01.STR.29.6.1122

[44] Charles Warlow and Wiley InterScience (Online service). 2008. Stroke : practical management. Blackwell Pub. 995 pages. https://www.wiley.com/en-ca/Stroke: +Practical+Management, +3rd+Edition-p-9781405127660

[45] C J Winstein, P S Pohl, C Cardinale, A Green, L Scholtz, and C S Waters. 1996. Learning a partial-weight-bearing skill: effectiveness of two forms of feedback. Physical Therapy 76, 9 (sep 1996), 985-993. http://www.ncbi.nlm.nih. gov/pubmed/8790276

[46] World Health Organization. 2011. WHO / World report on disability. Technical Report. http://www.who.int/disabilities/world\{_\}report/2011/en/

[47] Che Chang Yang and Yeh Liang Hsu. 2010. A review of accelerometry-based wearable motion detectors for physical activity monitoring. (2010), 7772-7788 pages. https://doi.org/10.3390/s100807772

[48] James W Youdas, Brian J Kotajarvi, Denny J Padgett, and Kenton R Kaufman. 2005. Partial weight-bearing gait using conventional assistive devices. Archives of Physical Medicine and Rehabilitation 86, 3 (2005), 394-398. https://doi.org/10. 1016/j.apmr.2004.03.026

[49] Lena Zidén, Margareta Kreuter, and Kerstin Frändin. 2010. Long-term effects of home rehabilitation after hip fracture - 1-year follow-up of functioning, balance confidence, and health-related quality of life in elderly people. Disability and Rehabilitation 32, 1 (2010), 18-32. https://doi.org/10.3109/09638280902980910 